\title{
CULTIVO DE CANNABIS Y DEGRADACIÓN DEL ESPACIO FORESTAL EN LA PROVINCIA DE TAOUNATE (RIF MERIDIONAL, MARRUECOS): REPERCUSIONES PAISAJISTICAS Y ECONÓMICAS
}

\author{
Enrique López Lara \\ Universidad de Sevilla \\ elopezl@us.es \\ Hassan Daïde \\ Mohamed Chaouki \\ Universidad Sidi Mohamed Ben Abdelah de Fez. Marruecos
}

\section{RESUMEN}

El cultivo del cannabis, consolidado en el Rif Central, ha experimentado un incremento, desde la década de los ochenta, tanto en su producción como en su extensión territorial, que se ha producido en dirección sur desde los núcleos originarios de Kétama y de Bab Berret, a costa de roturaciones de bosques. Se analiza la degradación del espacio forestal en el norte de la provincia de Taounate, mediante un estudio de los archivos judiciales en atención a las incidencias registradas por quema de la vegetación natural y un trabajo de campo que recoge las evidencias de estas roturaciones.

Palabras clave: Rif Meridional, cultivo del cannabis, degradación espacio forestal, repercusiones paisajísticas y socioeconómicas.

Fecha de recepción: marzo 2013.

Fecha de aceptación: enero 2014.

Trabajo producto del Proyecto de Investigación «El patrimonio paisajístico y cultural como recurso para el desarrollo territorial y local del norte de Marruecos: ejemplos de comunidades rurales del Rif y del Medio Atlas (Marruecos), financiado por la Agencia Española de Cooperación Internacional al desarrollo (AECId), entre la Universidad de Sevilla y la Universidad Sidi Mohamed Ben Abdellah de Fez. Referencias A/9760/07 y $\mathrm{A} / 016773 / 08$. 


\section{ABSTRACT}

The production and size of the cultivation of cannabis, a well-established practice in the Central Rif area, has increased since the eighties in the region southwards of Ketama and Bab Berret due to the plowing of forest areas. By studying the court records issued in response to the burning of wooded areas and the field work conducted to collect evidence of the subsequent farming of these areas, we are able to analyze the impact of the deterioration of the forest in the northern Taounate province.

Keywords: Southern Rif, cannabis cultivation, depleted forest areas, landscape and socio-economic impacts.

\section{INTRODUCCIÓN}

\section{I.1. Objetivo, área geográfica y período temporal del estudio}

Marruecos y, en concreto, su zona norte correspondiente al Rif, ha visto incrementar en los últimos decenios la extensión de la superficie dedicada al cultivo del cannabis y la producción de sus derivados (en especial, hachís), a pesar de las medidas tomadas para declarar su ilegalidad al final del reinado de Hassan II, en el año 1992. De hecho, estudios realizados por la Organización de las Naciones Unidas, Oficina contra la Droga y el Crimen (ONUDC, 2003, 2005, 2006 y 2007) en colaboración con el gobierno marroquí calcularon que, aproximadamente, en Marruecos se contabilizaban 134.000 hectáreas dedicadas a este tipo de cultivo en el año 2004, lo que le convierte en el mayor productor mundial (Chouvy, 2008), estando la mayor parte de ellas en el Rif (aproximadamente, el 90 \% total de la producción del país).

El Rif es un espacio montañoso del norte de Marruecos de accidentado relieve producto de su evolución y conformación geológica (El Abdellaoui y Chikhi, 1999). Ello, junto a posición y su condición geográfica, unido a su propia evolución histórica, donde destacan episodios que le han conferido un carácter independiente, han repercutido en un cierto aislamiento y marginalidad en el contexto geográfico, político y socio-económico. Carácter que ha sido reconocido recientemente por el propio monarca Mohamed VI quien puso en marcha medidas para superar estas dificultades (López Lara, 2000 y 2001).

El artículo analiza el incremento del cultivo del cannabis y el paralelo proceso de degradación de la vegetación forestal en la provincia de Taounate (Rif Meridional) y, en concreto, en su parte norte, entre las comunas de Ghafsai y Khlalfa, al sur de los núcleos tradicionales de cultivo del cannabis de Kétama y Bab Berret, en el Rif Central. Este estudio y sus análisis se han realizado mediante el desarrollo de un intenso trabajo de campo (realizado en dos periodos: septiembre-octubre de 2008 y abril-mayo de 2009) y la consulta de fuentes estadísticas diversas, en especial el registro de denuncias por quema ilegales existentes en los archivos judiciales de la provincia de Taounate.

La degradación de las condiciones naturales y ambientales junto a la pérdida de patrimonio natural (en especial, el representado por la riqueza forestal) están notablemente relacionadas con el incremento de la extensión de los terrenos dedicados al cultivo del cannabis. 
Asimismo, el incremento del cultivo del cannabis conlleva un crecimiento del nivel de vida de la población que a éste se dedica y, por ello, mayores expectativas en un medio hostil carente de auténticos proyectos de desarrollo, pese a los intentos realizados (López Lara, 2008). Este proceso posee, a la par, repercusiones o transformaciones socio-económicas y paisajísticas, que se ponen en evidencia en este artículo.

Geográficamente, el análisis se centra en las montañas de la parte meridional del Rif, las más bajas topográficamente, abarcando un espacio que se extiende aproximadamente sobre $3.400 \mathrm{~km}^{2}$, englobando la provincia de Taounate y, en concreto, el área donde su ubican las comunas de Ghafsai y Khlalfa. En éstas se desarrollan condiciones bioclimáticas particulares ya que posición geográfica y topografía otorgan influencias continentales, oceánicas y mediterráneas favorecedoras de la presencia de notables formaciones naturales de vegetación en forma de masas forestales, que vienen a representar un rico y variado patrimonio natural.

Sin embargo, en los últimos dos decenios, se viene padeciendo una notable degradación de sus espacios forestales que se explica, por un lado, por la antigüedad del poblamiento y la densidad de la población (por ejemplo, más de 200 hab./ $\mathrm{km}^{2}$ en la comuna de Bouadel), sin olvidar, por otro lado, el incremento y la presencia que va adquiriendo el cultivo del cannabis que ha acentuado de manera severa el proceso de degradación de las masas forestales y del patrimonio que representan.

\section{I.2. Estado de la cuestión}

En la evolución del conocimiento geográfico y territorial del Rif se pueden diferenciar tres fases secuenciadas y relacionadas con el desarrollo del contexto histórico, económico y político:

- Un primer período exploratorio, que se desarrolla desde finales del siglo XIX a principios del siglo XX, dominado por una geografía del descubrimiento que precedió y acompañó el comienzo de la etapa colonial y al Protectorado (Morales Lezcano, 1976).

- Un segundo período colonial que se corresponde con el Protectorado español (desde 1912 a 1956, aproximadamente) y con la colonización francesa allí donde correspondió, cuyo principal objetivo fue conseguir un conocimiento para la explotación económica de los recursos (El Abdellaoui y Chikhi, 1999) y la acción estratégica (Nogué y Vilanova, 1999).

- Con la Independencia, y desde los años sesenta, se desarrolla una tercera y novedosa fase, el período conocimiento universitario, especialmente fecundo desde la geografía (VV.AA., 2000), que se transformará con el paso de los años en período del conocimiento académico y científico de los territorios del Rif (López Lara, 2000).

Del Rif se han realizado estudios de calidad en esta última etapa universitario-académica, en su mayor parte en francés (VV.AA., 2000), -hecho que se explica por la formación predominante francesa de los geógrafos marroquíes- sobre diversos aspectos, bien en alguna de sus partes o en su totalidad, tales como su conformación geológica (Le Blanc, 1975; Asebriy, 1984; Bachnou, 1992), formaciones geomorfológicas (Maurer, 1968; Taouil, 1984; Tribak, 
1988; Laaouina, 1990; Driyef, 1994), condiciones climatológicas (Laadoua, 1987; Arraji, 1995), caracteres biogeográficos y edáficos (M’Hirit, 1982; Aalouane, 1988; El Alami, 1993), hidrología (Gartet, 1994; El Fengour y otros, 2009) y de preservación de bosques (Grovel, 1996).

También, aunque de menor cuantía se han abordado estudios sobre aspectos geográficos regionales y humanos, en especial los dedicados a la emigración (Bossard, 1988; El Hamraoui, 1984; Lazaar, 1989), al análisis regional (El Dahan, 1996; Sbai, 1990), a los caracteres rurales (Boudouah, 1985 y 2005; Yazami-Ztait, 1988; Ahmadan, 1991; Daïde, 2005), turísticos (Chaouki, 1994), urbanos (Ragala, 1987; Mohamer, 1994), históricos (Aziza, 1994), del género (Hajjarabi, 1987), aspectos lingüísticos (Chami, 1979; Hamdaoui, 1985), de la salud (Ouafiki, 1983 ; Bouziane, 1987; Ahrikat, 1985; Baroudi, 1993); y sociológicas (El Ouariachi, 1981; Jaydi, 1985).

Asimismo, existen estudios relacionados desde diversas perspectivas con la cuestión, como, por ejemplo, el de Zouaki (1984) sobre el uso epidemiológico de la utilización de la droga y el de Hajjaji, (1985) sobre el fenómeno del contrabando.

Se debe destacar la celebración de un Congreso en 1994 ¿Qué desarrollo para las montañas del Rif? (VV. AA., 1994) en el que se trataron aspectos relevantes para el desarrollo de este espacio, en la Universidad Abdelmalek Essadi (sede de Tetuán), de un Grupo de Investigación sobre el Rif, liderado por Mohamed Abdellaoui, contando con aportaciones internacionales (López Lara y Miranda, 1994).

Desde finales del siglo pasado existe una preocupación conjunta y compartida entre la ONU, el Gobierno de Marruecos y la Unión Europea por el crecimiento de las extensiones de terrenos dedicados al cultivo de cannabis y la consecuente producción de hachís en Marruecos, que se ha convertido en el principal proveedor de Europa de esta sustancia mediante el contrabando.

Se trata de un tema que se está abordando con más intensidad desde principios del nuevo siglo, en gran medida, por el incremento del fenómeno desde los años 80 y por las repercusiones económicas, sociales y ambientales que se derivan. En el estudio bibliográfico realizado para la confección de este artículo se detecta que la cuestión se aborda desde distintas perspectivas, tanto desde el punto de vista económico (por sus consecuencias internas, en forma de crecimiento económico y externas, por su relaciones con el contrabando y el tráfico de drogas) como desde el cultural (por la relevancia que adquiere su uso y consumo), pasando por las visiones política (acciones alternativas), geoestratégica (situación del Rif en el contexto del Estrecho de Gibraltar), ambiental (repercusiones en los cambios paisajísticos), etc.

Todas ellas son abordables desde planos o escalas diferentes: regiones del norte de Marruecos, espacio geográfico natural del Rif, área del Estrecho, contexto mediterráneo, Unión Europea, escenario internacional. La bibliografía consultada es sintomática de esta diversidad tanto en las perspectivas de análisis como en el tratamiento escalar.

Una de las primeras referencias encontradas sobre el cultivo del cannabis en el Rif es la aportación de Boudouah (1985), quien en su tesis de Tercer Ciclo aborda el tema del cultivo del kif y sus impactos económico y social en el Rif Central, aportando el caso de Kétama. Se apuntaba ya en este en este estudio pionero que la actividad económica derivada del cultivo del kif y su comercialización tenía una influencia capital sobre la comunidad rural, conociendo una transformación socio-económica y espacial sin precedentes que afectaba a una 
de las áreas más marginales de Marruecos. Con idéntica perspectiva, aunque extendiendo el ámbito territorial, aborda años más tarde Ahmadan (1991) la evolución del país Ghomara, como espacio rural periférico afectado por el cultivo del kif y su expansión, analizando la degradación ambiental.

Desde la perspectiva ambiental son escasas las aportaciones, entre las que cabe destacar la Tesis Doctoral de Moreno Torregrosa (1997), que realiza un excelente análisis del cultivo del cannabis en el Rif marroquí y sus consecuencias paisajísticas y ambientales. Son de especial reseña por su relevancia las aportaciones realizadas por Labrousse y Romero (2001 y 2002), con dos Informes sobre la situación del cannabis en el Rif marroquí del Observatorio Francés de Drogas y Toxicomanías. Del primero de los autores se debe un libro que desde las perspectivas geográfica y geopolítica aborda el análisis de las drogas a nivel mundial (Labrousse, 2004).

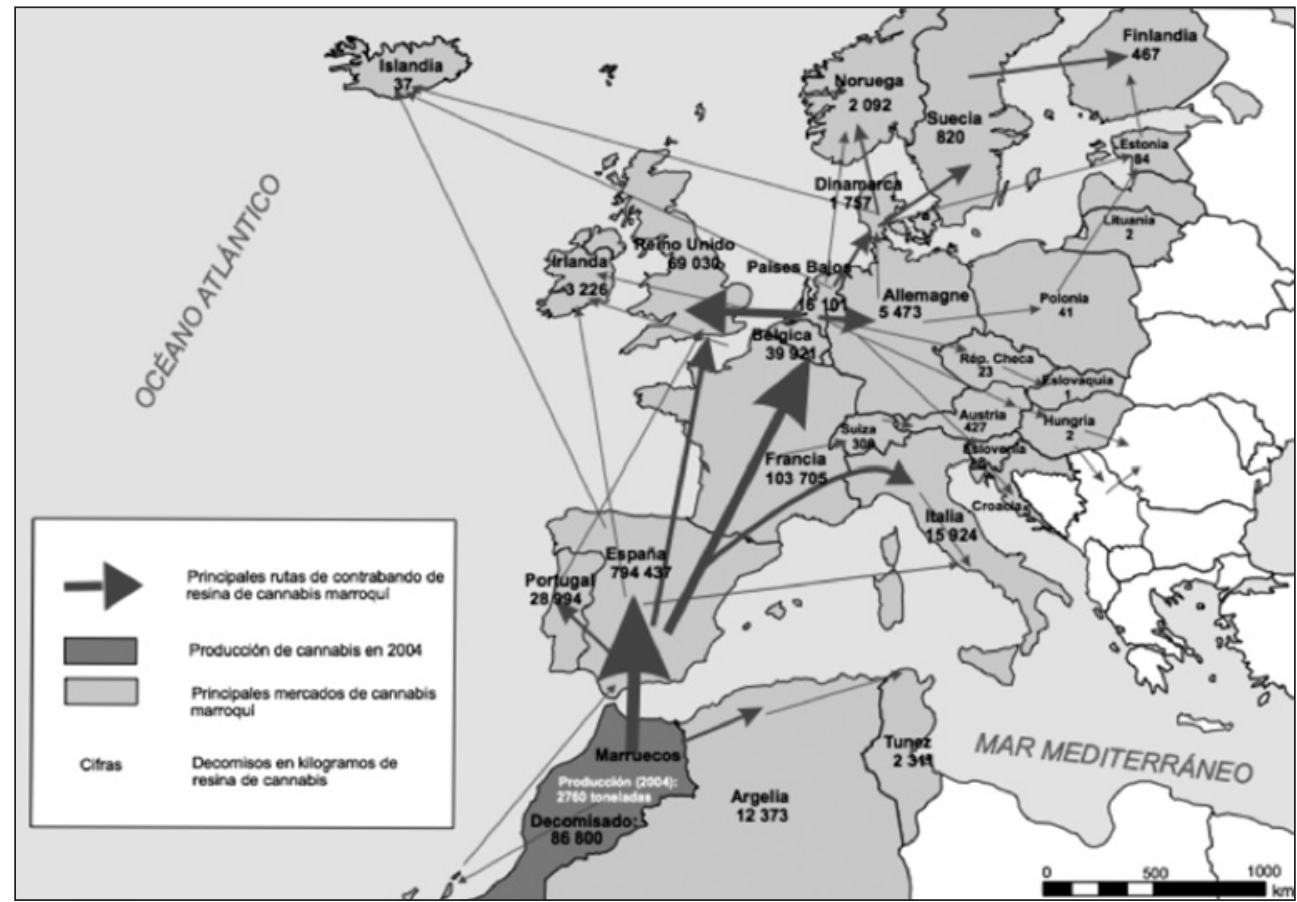

Fuente: elaboración propia a partir de ONUDC (2005).

De igual manera, Chouvy (2005 y 2008) analiza la cuestión desde la perspectiva geopolítica, relacionando el desarrollo de los cultivos de cannabis y el aumento del tráfico de drogas con el contexto de desarrollo institucional (normalmente de forma deslavazada e inconexa) y con las condicionantes geopolíticas (de manera más directa). En el mapa $n^{\circ} 1$ se observa la relevancia del tráfico de la resina de cannabis desde Marruecos con Europa. 
Desde el punto de vista social y sanitario, Sánchez y otros (2006), analizan los factores sociales, culturales, sanitarios y medioambientales que intervienen en el auge del cultivo del cannabis en el norte de Marruecos y los problemas para llevar a cabo un desarrollo local sostenible. El despegue económico que ha experimentado esta zona al amparo de la extensión de este cultivo ha tenido como consecuencia inmediata un incremento sustancial en el nivel de vida de la población local. Pace (2011) realiza un estudio de la interrelación entre procesos demográficos y la degradación del medio ambiente.

En el conocimiento de esta realidad tras la declaración de 1992 de ilegalización de los cultivos de cannabis en Marruecos, se realizan las encuestas sobre el cannabis en Marruecos (ONUDC, 2004, 2005 y 2007) que son de especial relevancia ya que nacen, como se ha apuntado, del consenso entre la ONU y el gobierno marroquí (Royaume du Maroc, 1994) por conocer la situación real del cultivo del cannabis. A través de estas encuestas y de un exhaustivo estudio de campo sobre el cultivo y la producción de kif en Marruecos, se radiografía la situación y se analizan las consecuencias de cara al tráfico de drogas hacia Europa (mapa n ${ }^{\circ}$ 1). Estos estudios se realizaron entre 2003 y 2007, sin que haya tenido continuación en los años siguientes. Sabir y otros (2008) publicaron el artículo más reciente sobre la extensión del cultivo del cannabis en relación a la problemática de la degradación de los recursos naturales en el Rif, en concreto, en su parte occidental.

Este artículo parte de lo anterior y viene a poner en evidencia un aspecto hasta ahora escasamente tratado, la expansión del cultivo del cannabis desde la zona tradicional, el Rif Central y Occidental (provincia de Chefchaouen), hacia el sur, el Rif Meridional (norte de la provincia de Taounate), analizando empíricamente el fenómeno y detallando las repercusiones ambientales y paisajísticas que trae la degradación debida a la deforestación y procesos de pérdida de suelo, a la par que se analizan estadísticas hasta ahora no contempladas, en especial los registros existentes en los archivos judiciales en relación a las quemas forestales ilegales.

\section{EL RECIENTE PROCESO DE DEGRADACIÓN DEL ESPACIO FORESTAL EN EL RIF MERI- DIONAL: NOROESTE DE LA PROVINCIA DE TAOUNATE}

La zona en estudio en esta aportación se corresponde con las bajas montañas del sur del Rif, que se extiende aproximadamente sobre $3.400 \mathrm{~km}^{2}$, y engloba el noroeste de la provincia de Taounate, en torno a las comunas de Ghafsai y Khalfa (mapa $\left.\mathrm{n}^{\circ} 2\right)$. El bosque de esta zona, fundamentalmente compuesto por diversas especies de encinas, se ubica sobre una extensión de aproximadamente 19.100 hectáreas, en las que se pueden censar aproximadamente 4.200 tipos de plantas aromáticas y medicinales en su denso matorral, que viene a ocupar 11.300 hectáreas, si bien, a título informativo, es de reseña que el espacio ocupado por el enebro apenas representa unas 70 hectáreas.

Se trata de una zona de expansión del cultivo y de la producción del cannabis. Anteriormente se centró, en los años 50, en el entorno de la carretera que une Chefchaouen con Targuist, en plena región Ghomara, en el Rif Central. A partir de los años 80, la expansión del cultivo del cannabis se fue desarrollando y expandiendo por el sur, en las bajas montañas del Rif, en la parte meridional de la provincia de Chefchaouen y en la septentrional de la provincia de Taounate y, en concreto, en el entorno de las comunas de Ghafsai y Khalfa. 
La distribución de especies vegetales en este espacio forestal al nor-oeste de Taounate permite establecer tres sectores geográficos diferenciados: zona del Jbel Oudka, zona del Alto Ouergha y zona del Ouergha Central, cuyas superficies varían entre las seis hectáreas del bosque Berhmoune en el municipio rural de Sidi El Mokhfi en la zona del Jbel Oudka y las 2.644 hectáreas del bosque Bourda en Fennaassa en el Alto Ouergha.

El fenómeno que se analiza en esta investigación es que, a pesar de las favorables condiciones climáticas, el espacio ocupado por bosque natural (e incluso las repoblaciones realizadas en los años 70) se encuentra en creciente regresión desde mediados del siglo XX, continuando en la actualidad.

En este sentido, las evidencias encontradas tanto en el estudio realizado a pie de campo como en el análisis de las estadísticas de la producción, permiten afirmar que la aparición del cultivo del cannabis es el principal causante del acelerado proceso de regresión y degradación de la vegetación natural, es decir, de las superficies forestales.

La cobertura vegetal de la zona en estudio se extiende sólo sobre unas 31.635 hectáreas, representando el bosque un 6\% en la zona de Ouergha Central, un 20\% en Alto Ouergha y un $25.5 \%$ en Jbel Oudka. Es de hacer notar que el porcentaje de superficie repoblada sólo supone el 0,7\% en el municipio de Galaz. Y si la cifra alcanza el 66\% en el municipio de Fennassa Bab El Hait en el Alto Ouergha, es prácticamente inexistente en los municipios de Sidi Yahya Bni Zeroual, Al Bibane y Sidi El haj M’hamed en el Ouergha Central.

\section{Mapa $n^{0} 2$}

CULTIVO DE CANNABIS EN LAS PROVINCIAS DEL NORTE DE MARRUECOS (2005)

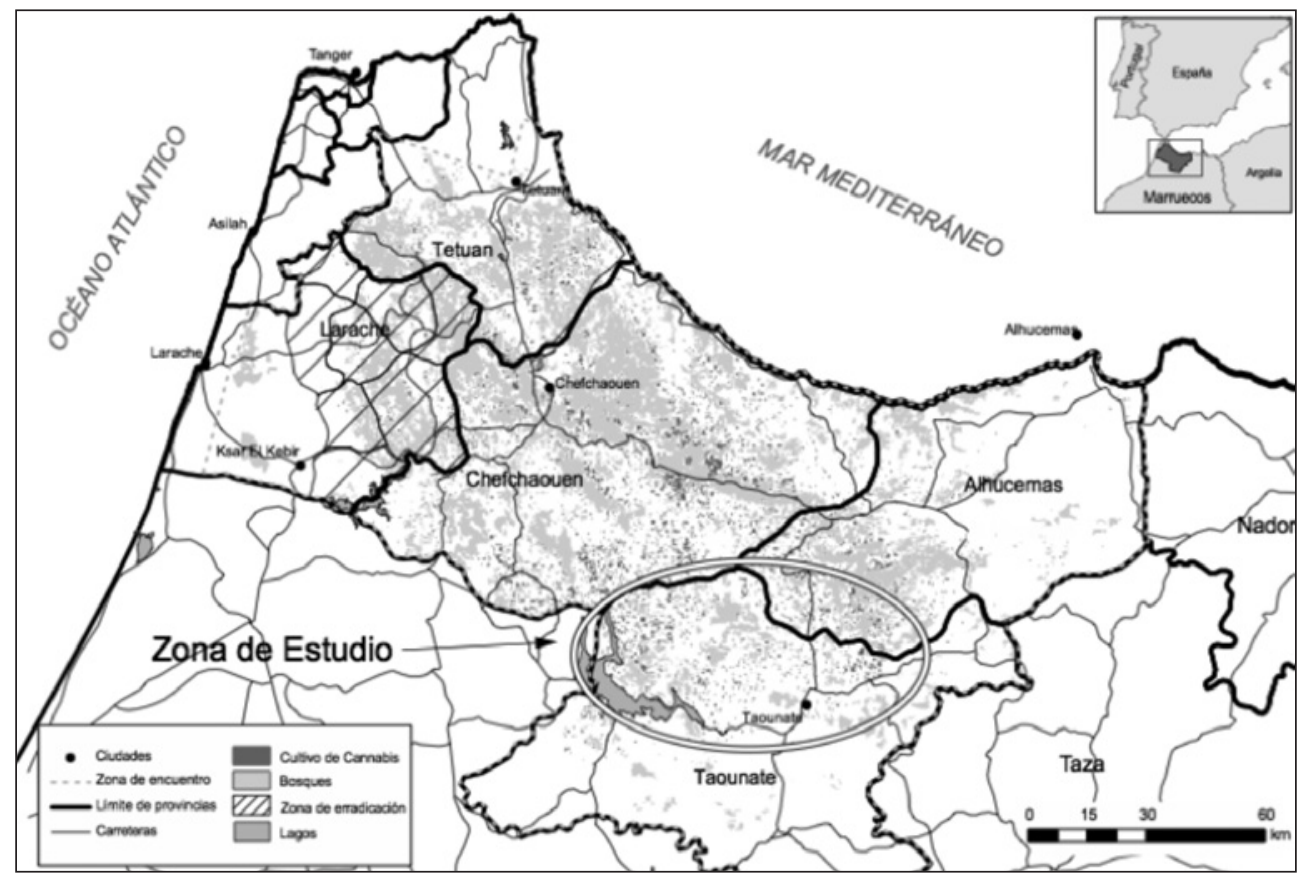

Fuente: elaboración propia a partir de ONUDC (2005). 
Estos porcentajes demuestran la intensidad y la envergadura de la degradación forestal existente en el sur del Rif. La antigüedad de la presencia humana en este espacio explica el estado y la situación de la cobertura vegetal ya que la presión de las actividades humanas en el bosque ha atendido, consuetudinariamente, a la satisfacción de las necesidades agrarias, pastoriles, domésticas e industriales. Es sintomático constatar que estos viejos bosques albergaban antes una fauna variada donde destacaban el león, el puerco espín y los monos. La desaparición de esta riqueza faunística atestigua un profundo desequilibrio del ecosistema regional.

Tradicionalmente, el modo de explotación en este espacio de montaña se basaba esencialmente en el pastoreo y en la agricultura. De hecho, el sobrepastoreo, debido a la existencia permanente de grandes rebaños de cabras en este espacio forestal, causó y aceleró el proceso de degradación de la vegetación natural. Es cierto que la vulnerabilidad del bosque es especialmente notable durante el período del brote (entre invierno y primavera) ya que las cabras arrasaban con éstos. No obstante, es de señalar que la sequía experimentada en esta área zona rifeña en la década de los años ochenta menguó el número de cabezas de ganado a casi la mitad (de 93.000 a 47.000 cabezas entre 1979 y 1994), proceso que se ha acentuado con la extensión del cultivo del kif, que ha reducido la cabaña de cabras a unas 35.000 cabezas en 2004 .

En esta zona el bosque es uno de los principales recursos para la alimentación del ganado, teniendo en cuenta que el proceso de engorde en los establos es prácticamente inexistente por la carencia de medios. La presión sobre el espacio se puede cuantificar en que en este espacio sólo se producen aproximadamente 220.000 unidades forrajeras mientras que las necesidades casi se triplican superan 620.000 unidades forrajeras.

\section{EL CRECIMIENTO DE LA SUPERFICIE DEDICADA AL CULTIVO DEL CANNABIS EN EL RIF CENTRAL Y SU EXPANSIÓN HACIA EL SUR}

Es de hacer notar que desde las primeras décadas del siglo XX el espacio forestal del sur del Rif Central experimentó un retroceso creciente. Según el nivel de regresión forestal, se pueden distinguir tres etapas en este proceso de deforestación:

- $\quad 1^{a}$ etapa: el período colonial (1912-1956). En esta etapa se redactó la primera ley relativa al patrimonio forestal en Marruecos. En 1917, la colonización francesa publicó el texto de Ley Relativa al Bosque. Esta Ley tuvo por objeto el establecimiento de una delimitación de uso de los espacios forestales. Cuestión que la población autóctona de la zona considerada no asimiló al entender que el bosque era un espacio de pasto colectivo que se había utilizado consuetudinariamente. Por lo tanto, los habitantes rifeños siguieron utilizando el bosque que degradaron con el aumento de la tala y la poda de ramas, fundamentalmente para la alimentación del ganado. Como consecuencia, durante la colonización se adoptó un sistema de trabajo oneroso para la población de esta zona del Rif que tenía un doble cometido: dominar la población allí asentada e impedir que afectaran al bosque además de contrarrestar el apoyo que la población le dio al movimiento de Abdelkarim El Khattabi durante la fase de su movimiento anticolonial y de liberación (1921-25). En 1956 el monarca Mohamed V, con la independencia, se resignó a tolerar la cultura del cannabis en los aduares históricos de Ketama, de Beni Seddate y de Beni Khaled. 
- 2 etapa: primer período de la independencia de 1956 a 1995. Durante esta etapa, el ritmo de degradación del bosque se ralentizó. Se realizaron esfuerzos mediante la repoblación de coníferas en el marco del Proyecto de Desarrollo Económico y Rural del Rif Occidental (D.E.R.R.O.), que empezó en el año 1961 (ONU/FAO) y vino a representar el primer proyecto de lucha contra la erosión y de mejora de las condiciones de vida, tras la independencia. Sin embargo, el proceso de degradación persistió en gran medida debido a que la actividad pastoril no encontró alternativa fuera del bosque. Las prácticas alimentarias tradicionales repercutieron en el bosque ya que la pobreza de los hogares, la alta densidad de población (alrededor de $100 \mathrm{ha} / \mathrm{km}^{2}$ ) y la falta de equipamientos alternativos (electricidad) fueron factores favorables a la degradación del bosque. Por otra parte, el recurso de gas butano estaba limitado y cuando existía, se utilizaba para el alumbrado de las casas. Incluso hoy en día la utilización de este gas dista mucho de estar generalizado en todos los hogares de los municipios rurales de Oudka, Tabouda, Ratba, Sidi El Haj Mohamed, Timezgana... La leña para la calefacción generalmente se obtiene del bosque que, consecuentemente, se va degradando.

- $3^{a}$ etapa: segundo período tras la independencia, desde 1996 a nuestros días. A partir de la región de Kétama, la superficie de cultivo del cannabis empieza a extenderse en como mancha de aceite hacia el sur desde el Rif Central. Esta expansión de la extensión se efectúa principalmente en detrimento de vegetación natural e incluso de las propias superficies que fueron repobladas, que no escapan a esta realidad.

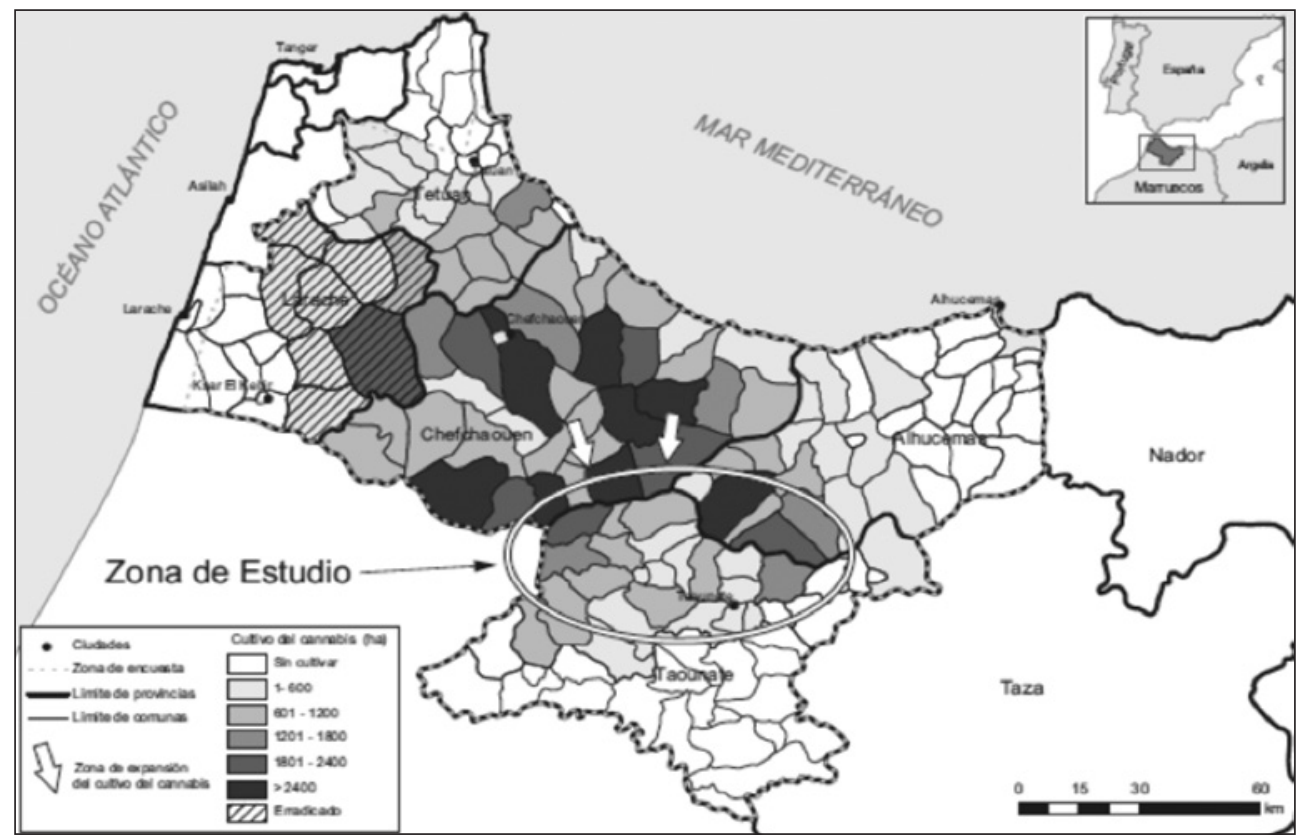

Fuente: elaboración propia a partir de ONUDC (2005). 
El espacio forestal de esta zona, en la fecha del estudio, ocupaba una superficie total de 36.535 hectáreas aunque se censaban unas 50.000 hectáreas en 1982, lo que representa una pérdida anual media de 650 hectáreas. La cubierta vegetal en la actualidad queda compuesta de la siguiente manera:

- bosque natural: 19.800 hectáreas,

- matorral: 7.200 hectáreas,

- superficie repoblada: 10.535 hectáreas (un 96\%, que se repoblaron con coníferas).

Destaca el hecho de que las superficies de repoblación, de escasa extensión territorial, se distribuyen en tres municipios rurales: Timezgna, Ain Mediouna y Tamedite, que suponen un $40 \%$ aproximadamente de la zona $\left(\right.$ mapa $^{\circ}{ }^{2}$ ). Es de hacer notar que algunos municipios rurales, como son los casos de An Bibane y de Sidi El Haj M’hammed, no se beneficiaron de la repoblación.

Como se ha comentado en el apartado anterior, la extensión del cultivo del cannabis es una realidad que se expande desde la zona central hacia el sur del Rif. Este fenómeno ha adquirido especial relevancia en gran medida por la impunidad con que las roturaciones se han producido y se producen. Cuestión que ha favorecido la intensidad de la degradación del bosque (cuadro $\mathrm{n}^{\mathrm{o}} 1$ ).

Cuadro $n^{\circ} 1$

EVOLUCIÓN DE LAS INFRACCIONES DEL DESMONTE FORESTAL

\begin{tabular}{|c|c|c|c|}
\hline Año & $\mathrm{N}^{\circ}$ pleitos por desmonte & $\mathrm{N}^{\circ}$ pleitos juzgados & $\%$ \\
\hline 1995 & 404 & 162 & 40,0 \\
\hline 1996 & 776 & 160 & 20,0 \\
\hline 1997 & 600 & 64 & 10,6 \\
\hline 1998 & 475 & 7 & 14,7 \\
\hline 1999 & 602 & 106 & 17,6 \\
\hline 2000 & 694 & 230 & 33,0 \\
\hline 2001 & 953 & 245 & 25,7 \\
\hline 2002 & 1316 & 328 & 25,0 \\
\hline 2003 & 808 & 801 & 99,0 \\
\hline 2004 & 970 & 475 & 49,0 \\
\hline 2005 & 966 & 362 & 37,4 \\
\hline 2006 & 618 & 544 & 88,0 \\
\hline 2007 & 555 & 422 & 70,0 \\
\hline Total & 9737 & 3906 & 40,0 \\
\hline
\end{tabular}

Fuente: Tribunal de Primera instancia de Taounate (2009).

No obstante, se ha observado un sensible aumento en el número de pleitos contra terceros responsables del desmonte forestal en relación, concretamente, con el cultivo del kif. Desde 1996, el número de pleitos no ha dejado de aumentar hasta alcanzar su máximo incremento en el año 2002, fecha en que este nuevo cultivo se extiende en la mayor parte de las explotaciones agrarias. 
El municipio rural de Khlalfa, próximo en dirección norte a la ciudad de Taounate (mapa $n^{\circ} 2$ ), es en este aspecto revelador: casi todos los agricultores se han reciclado al cultivo del cannabis.

También y fundamentalmente las roturaciones se deben a la lentitud judicial con la que se producen las emisiones de las condenas infligidas, cuando se producen. De hecho, como se comprueba en el cuadro $\mathrm{n}^{\circ} 1$, no se llegaron a condenar un $60 \%$ de las de roturaciones registradas entre los años 1995 y 2007. Síntoma de la permisividad de la administración competente hacia este tipo de prácticas ilegales.

\section{LA DEGRADACIÓN DEL ESPACIO FORESTAL Y EL INCREMENTO DEL CULTIVO DEL CAN- NABIS EN LAS COMUNAS DE GHAFSAI Y KHLALFA (NOROESTE DE LA PROVINCIA DE TAOUNATE)}

La extensión y la dirección que desarrolla el cultivo del cannabis y el incremento de los incendios forestales guardan una estrecha relación. En la comuna rural de Khlalfa, próxima a la ciudad de Taounate en dirección norte, el cultivo del cannabis no ha dejado de aumentar regularmente desde el año 1995, consecuencia directa del retroceso de las extensiones forestales y el incremento del número de incendios forestales acaecidos.

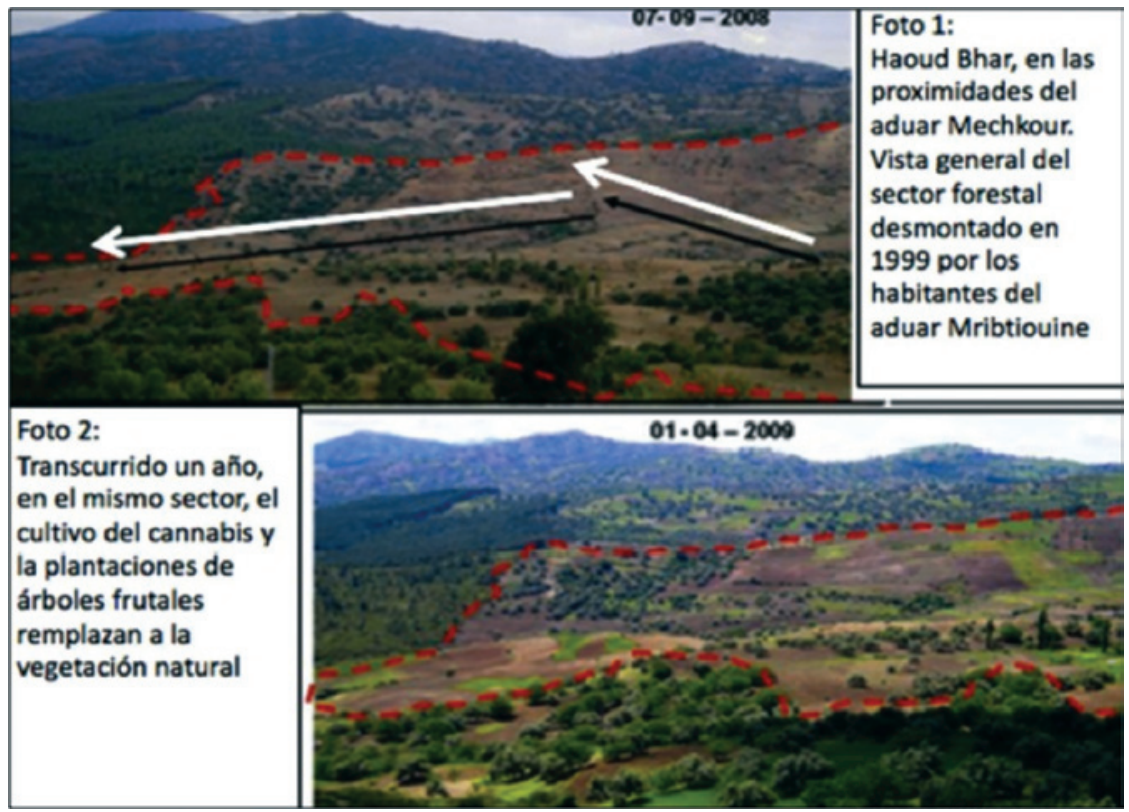

Foto 1: Haoud Bhar, en el aduar de Mechkour; vista general sector forestal desmontado en 1999 por los habitantes del aduar de Mribtiouine.

Foto 2: Transcurrido un año, en el mismo sector, el cultivo del kif y las plantaciones de árboles frutales remplazan la vegetación natural. 
En el año 2002 se registraba una superficie aproximada de 37 hectáreas de bosques quemados. En el periodo escaso de cinco años (1999 - 2003), la superficie forestal perdida por incendios superaba las 112 hectáreas en esta zona.

Entre los años 1998 y 2007 el número de las infracciones debidas al desmonte del bosque aumentó considerablemente, habiendo alcanzado 243 infracciones durante este período. La situación es más grave en los municipios rurales donde la repoblación ha sido más elevada, como es el caso de Ratba, que ha registrado un $36 \%$ de las infracciones durante este período.

Estas cifras demuestran la estrecha relación entre la extensión del cultivo del cannabis y la degradación del ecosistema de este medio montañoso, encontrándose los silvicultores sobrepasados ante la amplitud y la complejidad del fenómeno.

A finales de los años noventa, la expansión del desmonte no sólo afectaba al bosque autóctono sino que incluso se adentraba en las áreas forestales repobladas. De esta forma se ha destruido la mayor parte de los bosques de los municipios de Bab Trada, Bab Alaoua, Mgaz Tirane y Koudiat Lahcen, en el norte de la provincia de Taounate. Asimismo, el proceso de degradación ha alcanzado las coníferas y al matorral del sector occidental del mencionado bosque de Haoud Bhar, en el municipio rural de Khlalfa (gráfico ${ }^{\circ} 1$ ). En las fotos se puede observar la superficie forestal que ha sido desmontada por los habitantes del aduar Mribtiouine y cómo ese espacio, en un año, fue cultivado con plantaciones de cannabis.

El ritmo acelerado de la degradación del bosque, con el objeto de facilitar el cultivo del cannabis, causó, desde el año 2003, la desaparición en Bab Trada (monte Al Mencef) que constaba de una 449 hectáreas de encinas y alcornoques. Se trataba de un bosque antiguo que fue incendiado por los campesinos como reacción contra las autoridades locales que destruyeron todas las explotaciones existentes dedicadas al cultivo del cannabis. En el sector Mgaz Tirane, 135 hectáreas de roble y de plantas herbáceas fueron quemadas entre 2000 y 2004.

Existen otros factores directos, pero secundarios, que explican el proceso de degradación del bosque. Entre ellos están el pastoreo diurno o nocturno, la recogida de leña para calefacción y de madera para la industria artesanal y para la construcción de las casas. El hábitat rural presenta una relevante riqueza, destacando, en especial, las viviendas que conjugan, de manera equilibrada y armoniosa, materiales autóctonos, técnicas ancestrales de construcción y las condiciones del medio geográfico del entorno (López Lara y Obda, 2012). Es de hacer notar que los ingresos de la madera en Khlalfa (cuadro $\mathrm{n}^{\mathrm{o}} 2$ ) experimentan una notable regresión desde la aparición del cultivo del cannabis.

Cuadro $n^{0} 2$

INGRESOS PROCEDENTES DE LA EXPLOTACIÓN DE LOS RECURSOS FORESTALES EN EL MUNICIPIO RURAL DE KHLALFA

\begin{tabular}{|c|c|}
\hline Año & Ingresos en dírhams \\
\hline 1995 & 320.000 \\
\hline 2004 & 20.000 \\
\hline 2005 & 9.183 \\
\hline 2006 & 0 \\
\hline 2007 & 0 \\
\hline
\end{tabular}

Fuente: Eaux et Forêts, Taounate, 2009. 
El ámbito forestal, en el municipio Khlalfa, se encuentra delimitado vagamente por la administración (Ministère de l’Agriculture, 1995). Más relevancia adquiere el hecho de que la población autóctona ha reivindicado consuetudinariamente su derecho a la explotación del bosque. Esta situación, confusa, persiste hoy en día, a pesar o por la propia legislación forestal, que data, como se ha reseñado, del período colonial (año 1917). En el gráfico ${ }^{\circ} 2$ se puede observar las etapas de la degradación forestal en Bab Trada, bosque de la comuna de Khlalfa, que van desde la destrucción del bosque a la aparición del cultivo del cannabis.

Gráfico $n^{\circ} 2$

ETAPAS DE DEGRADACIÓN FORESTAL EN BAD TRADA (KHALFA)

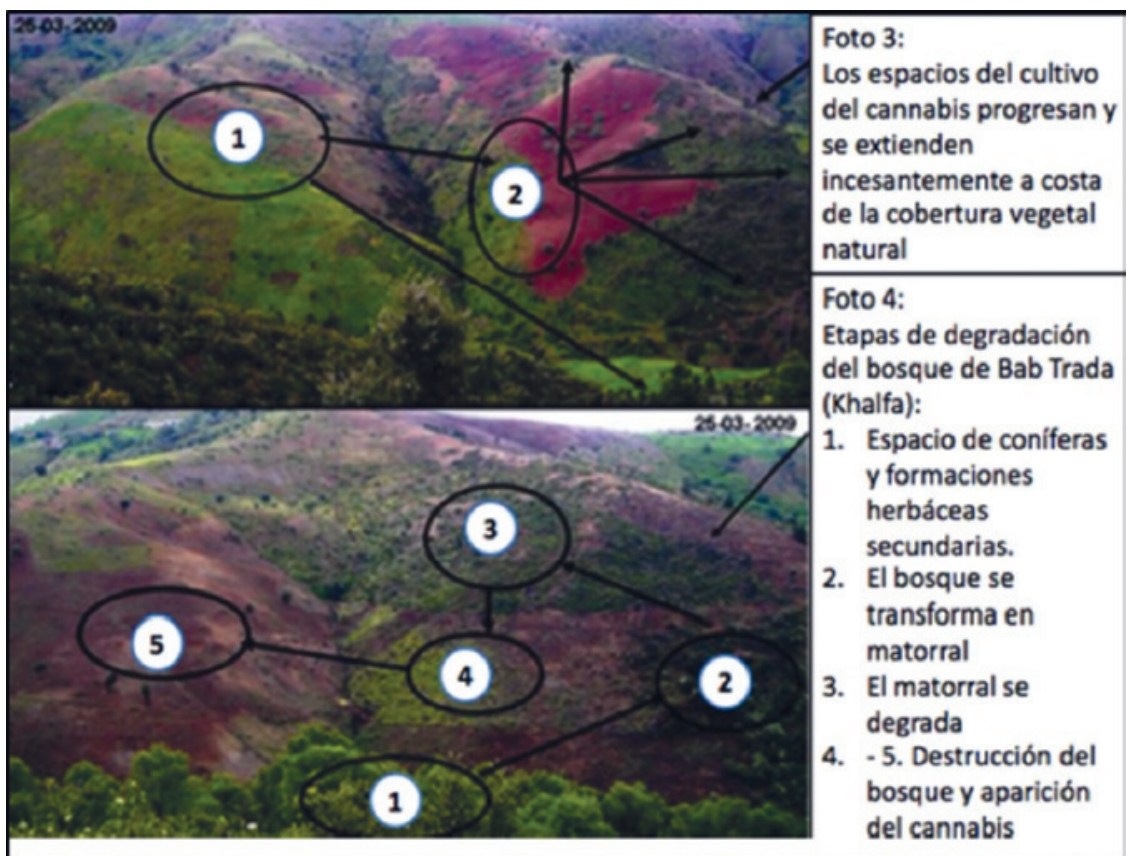

Foto 3: Los espacios del cultivo del cannabis progresan y se extienden incesantemente a costa de la cobertura vegetal natural.

Foto 4: Las etapas de la degradación del bosque en Bab Trada (Khlalfa). 1. Espacio de coníferas y de formaciones herbáceas secundarias. 2. El bosque se transforma en matorral. 3. La degradación del matorral. 4-5. Destrucción del bosque y aparición del cannabis

Como consecuencia, las relaciones entre los habitantes del municipio rural de Khlalfa y los guardas forestales, encargados de la conservación de los bosques, son frecuentemente tensas y conflictivas. El bosque es, para los primeros, un «espacio vital» al que creen tener derecho de explotación, máxime teniendo en consideración la insuficiencia de los recursos económicos locales.

Un estudio de Sabir y Roose (2004) sobre el contenido del carbono en los suelos dedicados al cultivo el cannabis y la estabilidad de los mismos, demuestra que el almacenamiento de las materias orgánicas difiere según los tipos de uso de los suelos (cuadro n 3 ). 
Cuadro $n^{0} 3$

EFECTOS DEL CULTIVO DEL CANNABIS SOBRE LAS CARACTERÍSTICAS DEL SUELO

\begin{tabular}{|c|c|}
\hline Tipo de usos del suelo & Velocidad de permeabilidad $(\mathrm{mm} / \mathrm{h})$ \\
\hline Bosque & 63.7 \\
\hline Matorral para pastoreo & 70 \\
\hline Cultivo de cannabis & 53 \\
\hline
\end{tabular}

Fuente: Sabir y Roose (2004)

De esta forma, los suelos de los bosques almacenan una gran cantidad de la materia orgánica, mientras que los suelos de matorral destinados al pastoreo pierden un $30 \%$ de materia prima. En cuanto a los suelos dedicados al cultivo del cannabis pueden llegar a perder hasta un $47 \%$ de sus materias orgánicas. Efectos que degradan las condiciones naturales de los espacios que paulatinamente se van roturando en beneficio del cultivo del cannabis.

\section{Gráfico n 3}

PROGRESO CULTIVO DEL CANNABIS EN LA MEDIA MONTAÑA DEL RIF MERIDIONAL

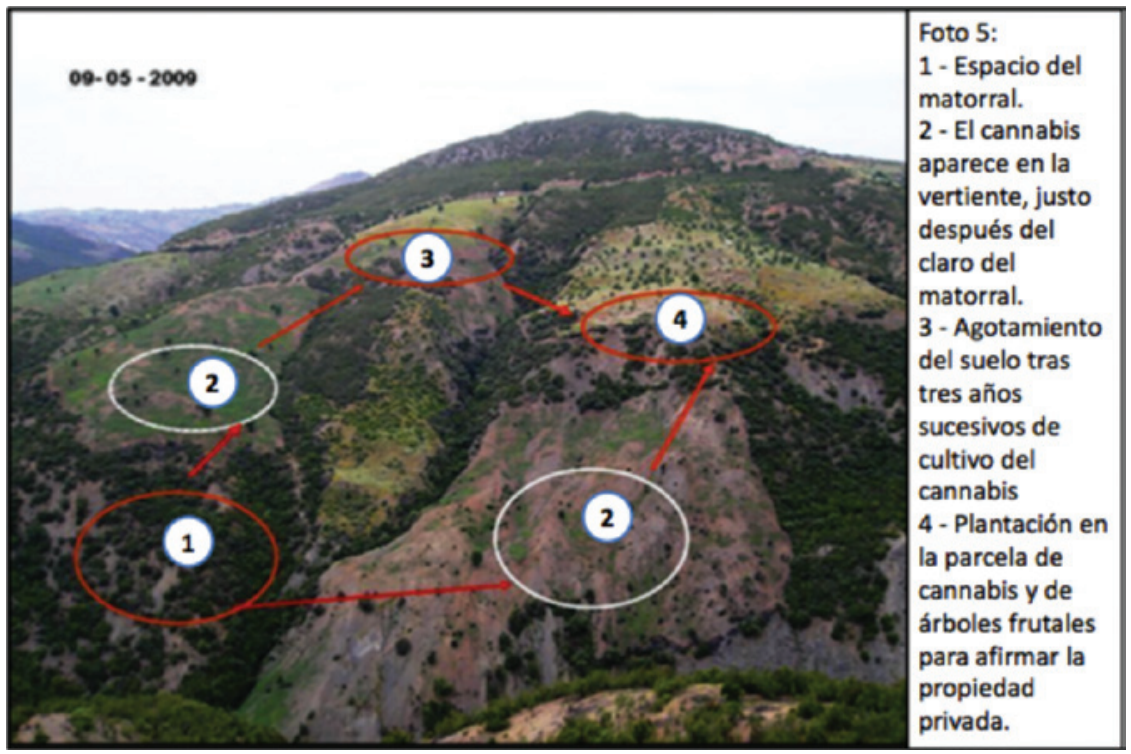

Foto 5; 1) Espacio del matorral. 2) El cannabis aparece sur la vertiente en cuesta, justo después del claro del matorral. 3) Agotamiento del suelo tras tres años sucesivos de cultivo de cannabis. 4) Plantación en la parcela del cannabis de árboles frutales para afirmar la propiedad privada. Prevalece todavía el cannabis.

En el caso de las explotaciones del cannabis especial relevancia adquiere la escorrentía superficial como factor empobrecedor, especialmente en las vertientes. De esta manera el empobrecimiento de las vertientes, muy notable en el municipio de Khlalfa (gráficos $n^{\circ} 2$ y 3 ), donde las vertientes oscilan entre los intervalos 15 y $25 \%$ y llegan a cubrir un $85 \%$ de la superficie global (Ministère de l'Agriculture, 1994). 
La degradación forestal en los cinco años anteriores al estudio alcanzaba una magnitud preocupante. La intervención de las autoridades locales en su lucha contra el cultivo del cannabis incitó a los campesinos a buscar otros espacios alternativos.

Las montañas del bajo Rif se han convertido, desde aproximadamente el año 1997, en el último refugio del cultivo del cannabis. Como consecuencia, se va destruyendo con rapidez la cubierta vegetal de esta zona geográfica sin ningún tipo de consideración. Los campesinos ven en su comportamiento una especie de compensación a la prohibición del cultivo del cannabis en las tierras más bajas.

\section{CONSIDERACIONES FINALES}

Los factores que explican el incremento de tierras dedicadas al cultivo del cannabis como alternativa económica importante y justificada para la población rural asentada al sur del Rif son múltiples y de distinta naturaleza, siendo dos de ellos relevantes. De un parte, las explotaciones agrarias dominadas por los cereales se convirtieron paulatinamente en escasamente rentables para los agricultores. Las explotaciones agrarias rifeñas, caracterizadas por el predominio de la micropropiedad, por el subempleo y por un desarrollo de técnicas ancestrales muy antiguas y escasamente tecnificadas, no resultan suficientes para alimentar a una población densa y en crecimiento. De otra parte, una comparación entre las ganancias generadas por el cultivo del cannabis y las que representan el cultivo de la cebada permiten explicar el incremento del cultivo del primero de ellos. Las ganancias brutas anuales del cannabis alcanzan 18.000 dirhams/por hectárea mientras que las de la cebada no sobrepasan 1.500 dirhams/ por hectárea, es decir, suponen un incremento del rendimiento económico 12 veces mayor. Por lo tanto, la prohibición del cultivo del cannabis en las explotaciones agrarias no puede ser eficaz sin una política económica global que concierte la diversificación de las posibilidades de empleo y la mejora de las condiciones de vida de la población de la zona.

Las repercusiones ambientales y paisajísticas de la roturación de los bosques y la expansión del cultivo del cannabis son evidentes. Suponen una pérdida del patrimonio forestal, un empobrecimiento del paisaje y un paulatino incremento de la erosión con la consecuente degradación de la riqueza de componentes de suelo.

Estas consideraciones se convierten en retos que ponen en evidencia la necesidad de soluciones, que pasan por la búsqueda de alternativas reales. Éstas deben de ser globales y no parciales, ya que sin visión de conjunto se pueden tomar medidas que en vez que detraer puedan reforzar la crisis del Rif.

Taiqui (1977) apuntaba tres objetivos principales que son relevantes en el diseño de un programa de desarrollo económico en el Rif. En primer lugar, la toma de medidas que frenaran la rápida expansión del cultivo del cannabis y el paralelo negocio relacionado con el contrabando. En segundo lugar, el establecimiento de una política de mejora e incremento de las producciones agro-silvo-pastoriles. Y, en tercer lugar, el desarrollo y la transferencia de conocimientos relativos a las interdependencias entre los sistemas humanos y naturales.

El cultivo del cannabis y su producción, que da lugar al contrabando, es una realidad en el Norte de Marruecos, en especial, en la zona del Rif, que hunde sus raíces en la tradición histórica y, más recientemente, en las concesiones otorgadas al Rif en el proceso de independencia de Marruecos. A pesar de que su abolición se decretó en el año 1992, poco antes del 
fallecimiento de Hassan II, ésta fue más nominal que real. La expansión del cultivo del kif en el Rif, en dirección sur, que ocupa numerosas comunas de las provincias de Chefchaouen y Taounate supone la base del crecimiento y sostén económico de los agricultores y habitantes rurales de una zona densamente poblada.

En este sentido cabe decir que existe un consenso tácito, un status quo, entre las autoridades y los agricultores que tiene sus repercusiones en forma de incremento económico y en forma de degradación del espacio natural. Se trata de un difícil equilibrio que da paso a la corrupción, al contrabando y a la marginación de la zona de los circuitos económicos oficiales que responde de forma inquietante a los desafíos que el desarrollo de las regiones del Norte de Marruecos necesita, auspiciado éste tanto por el propio gobierno de Marruecos como por Europa (López Lara, 2008; Iglesias, 2012).

Resulta imprescindible buscar alternativas de desarrollo compatibles con el medio, teniendo en cuenta que se trata de una de las zonas de mayor inmigración (frecuentemente a España) y más empobrecida del país marroquí.

\section{BIBLIOGRAFÍA}

AALOUANE, N. (1988): Caractéristiques édaphiques et bioclimatiques du massif forestier du Jbel Oudka (Rif Central, Maroc). Tesis Doctoral. Universidad de Estrasburgo I, Estrasburgo. Francia.

AHRIKAT, M. (1985): La situation sanitaire de la Province de Chefchaouen. Facultad de Medicina y Farmacia. Universidad Mohamed V, Rabat. Marruecos.

ALHAMA, J. y OTROS (2005): «Desarrollo humano sostenible: el ejemplo de la cultura del cannabis en el Rif (Norte de Marruecos)». Ecosistemas, 14.

AHMADAN, A. (1991): L'évolution récente d'un espace rural périphérique marocain : le pays Ghomara. UFR. Economía y Sociedad, Departamento Geografía. Universidad François Rabelais, Tours. Francia. 558 p.

ASEBRIY, L. (1984): Etude géologique de la zone subrifaine : nouvelle interprétation paléogéographique et structurale du Rif Externe (exemple du Moyen Ouergha). Facultad Ciencias, Universidad Mohamed V, Rabat, Marruecos; 187 p, 69 fig.

ARRAJI, M. (1995): Le climat du versant méditerranéen du Rif Central. Une géographie de la pluie : mécanismes pluviogènes et temps pluvieux. Universidad Toulouse-le-Mirail. Toulouse. Francia

AZIZA, M. (1994): Le Rif sous le protectorat espagnol : 1912-1956. Marginalisation et changements sociaux : naissance du salariat. Universidad Paris VIII. Paris. Francia, pp. 482.

BACHNOU, A. (1992): Stratigraphie et formes d'ammonites du Malm-Berriasien basal dans la région du Moyen Ouergha (Prérif Interne). Tesis doctoral. Universidad Claude Bernard (Lyon), France. 196 p., 50 tabl., 58 fig.

BAROUDI, F. (1993): L'approvisionnement en eau potable et les maladies hydriques de Taounate. Facultad de Medicina y Farmacia. Universidad Mohamed V, Rabat. Marruecos.

BORDES, P. y LABROUSSE, A. (2004): Économie de la drogue et réseaux de corruption au Maroc, Politique Africaine, $\mathrm{n}^{\circ}$ 93, p. 63-82. 
BOUDOUAH, M. (1985): La culture du kif et son impact économique et social dans le Rif Central. Tesis Tercer Ciclo, Geografía. Universidad de Toulouse II.

BOUDOUAH, M. (2005): Evolution de l'économie rurale, ses différentes implications et perspectives du développement dans le Rif Central-Maroc, Universidad de Chérif Idrisi. Marruecos.

BOUZIANE, A. (1987): La situation sanitaire de la Province de Taounate . Instituto Agronómico y Veterinario Hassan II, Rabat, Marruecos.

BOSSARD, R. (1988): Mouvements migratoires dans le Rif Oriental: le travail en Europe. Aspects contemporains majeurs des migrations dans la province de Nador. Universidad Paul Valery (Montpellier III). Montpelier, Francia. pp. 213

CHAMI, M. (1979): Un parler amazigh du Rif marocain: approche phonologique et morphologique. Universidad René Descartes, Paris V, Paris. Francia. 424 p.

CHAOUKI, M. (1994): Le tourisme dans les médinas de l'extrême Nord-Ouest marocain . UFR Ciencias. Geografía y Ordenación. Universidad de La Provenza (Aix-Marsella I). Aix-en-Provence, Francia.

CHOUVY, P.A. (2005): Raquettes de contrebande au Maroc: le haschisch, des persones et la contrebande. Jane Renseignement, vol 17, $\mathrm{n}^{\circ} 12$ pp. 40-43

CHOUVY, P. A. (2008): Production de cannabis et de haschich au Maroc : contexte et enjeux» Espace Politique : revue en ligne de géographie politique et de géopolitique, 4, p. $5-19$

DAÏDE, H. (2005): Espace et société au sud du Rif Central. Tesis Doctoral, Facultad de Letras y Ciencias humanas de Fez, 517 p.

DRIYEF, M. (1994): Les rides prérifaines et leurs bordures: étude géomorphologique et cartographique. Universidad de Toulouse-le-Mirail. Toulouse. Francia, 350 p.

EL ABDELLAOUI, M. y CHIKHI, N.E. (1999): «Explotación de recursos naturales». España en Marruecos. Edit. Milenio. pp. 509-550

EL ALAMI, R. (1993): Phytoclimatologie de la montagne marocaine: le Rif Central. Universidad de Paris VII. Paris, Francia. pp 178

EL DAHAN, M. (1996): Chefchaouen et sa région. Facultad de Derecho, Economía y Ciencias Sociales. Universidad François Rabelais, Tours, Francia 445 p.

EL HAMRAOUI, A. (1984): Le phénomène migratoire et son impact sur la société et l'espace dans le Rif Instituto Geográfico Daniel Faucher. Univ. Toulouse-le-Mirail (Toulouse II) Toulouse, Francia, pp. 386.

EL FENGOUR, M. y OTROS (2009): «Dégradation de la qualité des eaux du Barrage Sahla: traitement et gestion des risques de pollution (Province de Taounate, Rif-Maroc Septentrional)». Papeles de Geografía, nº 49-50, pp. 41-54.

EL OUARIACHI, K. (1981): Le Rif Oriental: transformation sociales et réalités urbaines. EHESS Univ. Panthéon-Sorbonne, Paris I, Paris, Francia 440 p.

GARTET, A. (1994): Morphogenèse et hydrologie dans le bassin-versant de l'oued Lében (Rif Méridional et Prérif et Oriental). UFR Geografía y Ordenación del territorio. Instituto Geográfico. Universidad de Provence (Aix-Marsella I) Francia. pp. 493

GROVEL, R. (1996): «La préservation des forêts du Rif centro-occidental: un enjeu de développement de la montagne rifaine», Revue de Géographie Alpine, n 4, p. 75-94.

HAJJAJI, T. (1985): Le phénomène de la contrebande au Maroc. ENAP. Rabat, Marruecos. 
HAJJARABI, F. (1987): Les souks féminins du Rif Central. Anthropologie de l'échange féminin Universidad Paris VII, Paris, Francia

HAMDAOUI, M. (1985): Description phonétique et phonologique d'un parler amazigh du Rif marocain (Province d'Al hoceima). Universidad Aix-Marsella I, Aix-en-Provence, Francia. 407 pp.

IGLESIAS, M. (2011): La cooperación transfronteriza Andalucía - Norte de Marruecos. Balance y perspectivas. Consejería de Presidencia. Junta de Andalucía. Sevilla. pp. 230.

JAYDI, N. (1985): Transformations économiques et structures familiales : le cas d'une vallée du Rif central: les Béni Boufrah. Universidad de Toulouse, Toulouse. Francia.

LAADOUA, A. (1987): Les variations spatio-temporelles des précipitations au Maroc Septentrional. UFR Geografía. Univ \& TTech. Flandres Artois (Lille I) Lille, Francia.

LAAOUINA, A. (1990): Le Maroc Nor-Oriental : reliefs, modèles et dynamique du calcaire. Publicaciones del Rectorado de la Universidad Mohamed I, Oujda, Marruecos.

LABROUSSE, A. (2004): Géopolitique des drogues, Presses Universitaires de France, coll. Que sais-je ? no 3693. Paris. Francia.

LABROUSSE, A. y ROMERO, L. (2001): Rapport sur la situation du cannabis dans le Rif marocain. Observatoire français des Drogues et des toxicomanies (OFDT).

LABROUSSE, A. y ROMERO, L. (2002): Maroc: la production de cannabis dans le Rif. Drogues Trafic International, Observatoire français des drogues et toxicomanies (OFDT), $n^{\circ} 13$.

LAZAAR, M. (1989): La migration internationale de travail et ses effets sur les campagnes du Rif (Province d'Al hoceima). Departamento Geografía. UFR Ciencias Humanas, Universidad de Poitiers, Francia. 571 p.

LE BLANC, D. (1975): Etude géologique dans le Rif externe Oriental au Nord de Taza. Edit. Laboratorio Geología Mediterráneo. Toulouse, France. 193 p. 50 fig.

LÓPEZ LARA, E. (2000): «Estudio de las aportaciones académico-científicas europeas al conocimiento geográfico de la región norte de Marruecos». Hacia un nuevo espacio euromediterráneo. III ${ }^{\circ}$ Encuentro de Geografía Regional. Barcelona. pp. 328-333.

LÓPEZ LARA, E. (2001): «Diagnóstico territorial de las montañas del Rif (Marruecos). Propuesta de desarrollo territorial de un espacio subdesarrollado». Actas del XVII Congreso de Geógrafos Españoles. Forma y función del territorio. Universidad de Oviedo, CeCodet, AGE y GEA. Oviedo. pp. 381-383.

LÓPEZ LARA, E. (2005): «La imagen turística de Marruecos proporcionada por Internet: reinterpretando el orientalismo». Cuadernos de Turismo, 16. Murcia pp. 123-134.

LÓPEZ LARA, E. (2008): «Cooperación y desarrollo territorial en el espacio fronterizo del norte de Marruecos». Estudios Fronterizos, 17. México. pp. 43-71.

LOPEZ LARA, E. y MIRANDA BONILLA, J. (1994): «L'aménagement du territoire et alternatives de développement dans les espaces naturels: l'expérience andalouse». Développement des montagnes rifaines: Quelle stratégie? Universidad Abdelmalek Essaâdi. Publications de la Faculté des Lettres et des Sciences Humaines. Colloques, 6. Tetuán, Marruecos. pp. 293-300.

LÓPEZ LARA, E. y OBDA, Kh. (2012): «Estudio de casos de hábitat rural en las montañas del Medio Atlas y del Rif (Marruecos)». Anales de Geografía de la Universidad Complutense de Madrid, $\mathrm{n}^{\circ} 32$, pp. 29-43. 
MAURER, G. (1968): «Les Montagnes du Rif Central : Etude géomorphologique». Travaux Scient. , Série géologie et géographie physique, $\mathrm{n}^{\circ} 14$. Rabat, $499 \mathrm{p}$.

M’HIRIT, O. (1982): «Etude écologique et forestière de cédraies du Rif marocain. Essai sur une approche multidimensionnelle de la phyto-écologie et de la productivité du cèdre». Annal. Rech. Forest, tome n ${ }^{\circ} 22$ Rabat. Marruecos, pp. 502.

MINISTÈRE DE L'AGRICULTURE, (1994): Plan d'aménagement anti-érosif du bassin versant de l'oued Ouergha en Amont du barrage Al Wahda. Rapport principal. Admon. des Eaux et Forêts et de la Conservation du Sol. Rabat.

MINISTERE DE L'AGRICULTURE, (1995): Stratégie de développement des terres de parcours au Maroc. Rabat.

MORALES LEZCANO, V. (1976): El colonialismo hispano-francés en Marruecos (18981927). Madrid. S. XXI.

MORENO TORREGROSA, P. (1997): Estudio del cultivo del cannabis sativa en el Rif marroquí: sus consecuencias socioeconómicas para la región. Tesis Doctoral. Escuela Técnica Superior de Ingenieros Agrónomos. Universidad Politécnica de Valencia. pp. 320.

NOGUÉ, J. y VILANOVA, J.L. (1999): España en Marruecos. Edit. Milenio. pp. 570.

ONUDC (2007) Office contre la drogue et le crime et A.P.D.E.S.P.N.M . Maroc, enquête sur le cannabis, 2003, 2005, 2006 y 2007. Vienne.

PACE, R. (2001): «Interrelación entre procesos demográficos y degradación del medio ambiente en un área de alto riesgo». Cuadernos Geográficos de la Universidad de Granada, $\mathrm{n}^{\mathrm{o}} 31 \mathrm{pp} .23-52$.

ROYAUME DU MAROC, (1994): Livre Blanc. La politique genérale du Maroc dans le domaine de lutte contre les drogues et pour le développement économique des provinces du Nord. Versión árabe, 149 p.

SABIR, M. y ROOSE, E. (2004): «Influences du couvert végétal et des sols sur le stock de carbone du sol et les risques d'érosion et de ruissellement dans les Montagnes Méditerranéennes du Rif occidental». Actes des JSIRAUF. In Bulletin réseau érosion $\mathrm{n}^{\circ} 23$, Montpellier.

SABIR, M. y OTROS (2008): «Extension de la culture du cannabis et problématique de dégradation des ressources naturelles au Rif occidentale». $R G M, \mathrm{n}^{\circ} 1-2$, février 2008 , nouvelle série, pp.81-93.

SAKROUHI, A. (1982): «Logique économique paysanne et logique du capital. Étude de la dynamique sociale dans une région périphérique : le cas du Rif de 1960 à 1980». Universidad Paul Sabatier (Toulouse I) Toulouse, Francia

SÁNCHEZ, J. y OTROS (2006): «Estructuras sociales de desigualdad, medio ambiente y desarrollo humano: cannabis y desarrollo en Marruecos». Portularia, VI. Huelva, pp. 105-121.

SBAI, A. (1990): «L'organisation de l'espace dans la Province de Taounate». Universidad de Lyon II, Lyon, France.

TAIQUI, L. (1997): «La dégradation écologique au Rif marocain : nécessités d'une nouvelle approche». Mediterranea, 17. Serie de Estudios Biológicos. pp. 5-17.

TAOUIL, A. (1984): «L'érosion dans le Haut Rhis (Targuist, Rif Central): étude socio-écologique». Instituto de Geografía. Universidad de Toulouse-le-Mirail (Toulouse II). Francia. 320 p. 
TRIBAK, A. (1988): «L'érosion du Prérif Oriental: contribution à l'étude de la dynamique actuelle dans quelques bassins du Nord de Taza». Instituto de Geografía Alpina. Universidad Joseph Fourier (Grenoble I), Grenoble. Francia. 258 p.

VV.AA. (1994): Développement des montagnes rifaines: Quelle stratégie?. Universidad Abdelmalek Essaâdi. Publicaciones de la Facultad de Letras y Ciencias Humanas, ${ }^{\circ} 6$. Tetuán, Marruecos. pp. 293-300.

VV.AA. (2000): Répertoire des Thèses Universitaires réalisées sur le Rif (Maroc). Groupe de Recherches Géographiques sur le Rif. Publicaciones de la Facultad de Letras y Ciencias Humanas. Tetuán. Marruecos. 328 p.

YAZAMI-ZTAIT, M. (1988): Le D.E.R.R.O., vingt-cinq ans après: milieux naturels et aménagement à Taounate. Instituto de Geografía Daniel Faucher. Universidad Toulouse-leMirail (Toulouse II) Toulouse, Francia.

ZOUAKI, A. (1984): Contribution à l'étude épidémiologique de l'usage de drogue à Tétouan. Facultad de Medicina y Farmacia. Universidad Mohamed V, Rabat. Marruecos. 